\title{
Retraction of: A Comparative Study of ARHI Imprinted Gene Detection and Fine-Needle Aspiration Cytology in the Differential Diagnosis of Benign and Malignant Thyroid Nodules by Dazhong Zou, et al., Genet Test Mol Biomarkers 2019;23(9):681-687; Doi: $10.1089 / \mathrm{gtmb} .2019 .0028$
}

The Editor of Genetic Testing and Molecular Biomarkers officially retracts the article entitled, A Comparative Study of ARHI Imprinted Gene Detection and Fine-Needle Aspiration Cytology in the Differential Diagnosis of Benign and Malignant Thyroid Nodules, by Dazhong Zou, et al. (Genet Test Mol Biomarkers 2019;23(9):681-687; doi: 10.1089/gtmb.2019.0028) based on the communication received from the corresponding author, reproduced here as it was written:

It is with deep regret that I write to inform you about our request for the withdrawal of our submitted article entitled "A Comparative Study of ARHI Imprinted Gene Detection and Fine-Needle Aspiration Cytology in the Differential Diagnosis of Benign and Malignant Thyroid Nodules" published in the Genetic and Molecular Biomarkers Journal (Volume 23, Number 9, 2019, Pp. 681-687) for the following reason: By the time we submitted this paper, we did not have proper communication with Lisen Imprinting Diagnostics which conducted the experiment for us. So, we had some misunderstanding of the important information for writing the paper. The ARHI gene that we claimed to be a thyroid cancer biomarker is an inactivated thyroid cancer imprinting gene. Therefore, the image presented in Figure 2 of the paper was invalid. We are deeply sorry to the editor and the review board for these errors in judgment. and for undermining the journal's integrity. We are willing to accept the intended penalties the journal find appropriate. Thank you for your consideration.

Sincerely,

Dazhong Zou

By definition, authors are fully responsible for the content of their articles. As such, the Editor and Publisher of Genetic Testing and Molecular Biomarkers find this to be a significant violation of all standard and proper protocols of scientific reporting and, as such, officially retract this paper from the literature. The Editor also reported this infringement to the department chairs of the authors' institutions.

Genetic Testing and Molecular Biomarkers is committed to upholding and protecting the scientific record and the community it serves. 